\title{
Different clinical effect of 4 anti-dementia drugs for Alzheimer's patients depending on white matter severity
}

Yusuke Fukui, Nozomi Hishikawa, Jin Ichinose, Kota Sato, Yumiko Nakano, Ryuta Morihara, Yasuyuki Ohta, Toru Yamashita, and Koji Abe*

Department of Neurology, Graduate School of Medicine, Dentistry and Pharmaceutical Sciences, Okayama University, 2-5-1 Shikatacho, Kitaku, Okayama 700-8558, Japan

*Corresponding author: Prof. Koji Abe

Department of Neurology, Graduate School of Medicine, Dentistry and Pharmaceutical Sciences, Okayama University, 2-5-1 Shikata-cho, Okayama 700-8558, Japan.

Tel.: +81-86-235-7365, Fax: +81-86-235-7368, E-mail: peer1tcc@s.okayama-u.ac.jp

Short running title: Four anti-dementia drugs for AD with WMLs 


\begin{abstract}
AIM: In order to examine the clinical effect of four anti-dementia drugs (donepezil, galantamine, rivastigmine, and memantine) in Alzheimer's disease (AD) patients who were divided into subgroups based on their periventricular hyperintensity $(\mathrm{PVH})$-severity.
\end{abstract}

METHODS: 551 AD patients (201 males and 350 females) were divided into four subgroups based on their PVH-severity (0-III). They received monotherapy for 12 months (M). We compared the clinical effects at the baseline and at 3,6, and $12 \mathrm{M}$ after initiation.

RESULTS: The baseline age became higher with PVH grades, and the mini-mental state examination (MMSE) and Hasegawa dementia scale-revised (HDS-R) showed a decrease that was dependent on white matter (WM) severity. Although the PVH 0 subgroup showed stable cognitive, affective, and ADL functions until $12 \mathrm{M}$ in all four drug groups, the PVH I subgroup showed an improved apathy scale (AS) from the baseline in response to memantine at 3 and $9 \mathrm{M}(\mathrm{p}<0.05)$, and galantamine at 9 M ( $p<0.01)$. In the PVH II subgroup, MMSE showed a significant improvement from the baseline in response to galantamine $(\mathrm{p}<0.05)$ at 9 M and HDS-R $(\mathrm{p}<0.05)$ at $3 \mathrm{M}$. In the PVH III subgroup, cognitive and affective functions were preserved in all four drug groups until $12 \mathrm{M}$, but ADL deteriorated in the riverstigmine group at 6 and $12 \mathrm{M}(\mathrm{p}<0.05)$. 
CONCLUSIONS: The present study demonstrates that these four drugs showed sensitivity dependent on WM severity that clinically affected cognitive, affective and ADL functions.

Keywords: Alzheimer's disease, anti-dementia drug, magnetic resonance imaging, periventricular hyperintensity, white matter lesions

\section{Introduction}

As a result of a rapidly aging world population, dementia has become a significant social priority. Alzheimer's disease (AD) is the most common cause of dementia, and is a neurodegenerative disease characterized by the presence of amyloid-beta $(\mathrm{A} \beta)$ plaques with neuritic plaques and neurofibrillary tangles that add to the severity of these changes ${ }^{1}$.

White matter lesions (WMLs), including periventricular hyperintensity (PVH) and deep white matter hyperintensity (DWMH), are visualized as an increased signal on T2-weighted and fluid-attenuated inversion recovery (FLAIR) magnetic resonance imaging (MRI) sequences ${ }^{2}$, and are observed in normal elderly adults as well as in AD patients. In a previous report, WMLs are associated with clinical risk and a symptomatic course of $\mathrm{AD}^{3}$. The volume of PVH was also associated with neurofibrillary tangles and the volume of DWMH was associated with diffuse plaques indicating that AD pathology may contribute to the vascular or white matter pathology ${ }^{4}$. Notably, our previous studies demonstrated that the severity of WMLs was closely related to AD pathology, and strongly 
influenced the deterioration of cognitive and affective functions in AD patients ${ }^{5,6}$. Early stage AD patients may be more vulnerable to the cognitive effect of WHLs than cognitively normal elderly adults with a similar burden of WHLs ${ }^{7}$.

Although there has been much debate about the relation between AD pathology and WMLs, there is no report on the WML-dependent effectiveness of drugs on AD patients. In the present study, therefore, we examined the clinical effect of four anti-dementia drugs (donepezil, galantamine, rivastigmine, and memantine) in AD patients who were divided into subgroups based on their PVH severity.

\section{Materials and Methods}

\section{Participants and study design}

This was a retrospective clinical cohort study performed in the outpatient clinic of

Okayama University Hospital and affiliated hospitals from June 2011 to March 2016. For the present study, we recruited 551 AD patients (201 males and 350 females; $78.4 \pm 7.3$ years old, mean \pm SD) that were on monotherapy with donepezil, galantamine, rivastigmine, or memantine. All patients are prescribed anti-AD drugs for the first time. Those patients that were receiving combination therapy or 
that switched to a combination therapy of cholinesterase inhibitor plus memantine were excluded from this study. AD patients were diagnosed with probable or possible AD according to the National Institute of Neurological and Communicative Disorders and Stroke AD and Related Disorders Association (NINCDS-ADRDA). Magnetic resonance imaging (MRI) was used to estimate changes in white matter (WM) with PVH using the Fazekas scale ${ }^{8}$ on T2 weighted images (T2WI) and fluid attenuated inversion recovery (FLAIR) images, and were divided into four subgroups according to the grade of PVH (0-III). Furthermore, parahippocampal gyrus atrophy of each PVH group was evaluated using Z score of the voxel-based specific regional analysis system for Alzheimer's disease (VSRAD) ${ }^{9}$ which become an indicator reflecting the degree of the entorhinal cortex for diagnosis of early $\mathrm{AD}^{10}$. The drug dose was gradually increased as follows: donepezil, from 3 to 5 or $10 \mathrm{mg}$; galantamine, from 8 to 16 or $24 \mathrm{mg}$ over 4 or 8 weeks; rivastigmine, from 4.5 to $18 \mathrm{mg}$ over 16 weeks; memantine, from 5 to $20 \mathrm{mg}$ over 4 weeks. Cognitive functions were assessed using the mini-mental state examination (MMSE) and Hasegawa dementia scale-revised (HDS-R). The behavioral and psychological symptoms of dementia (BPSD) were assessed using the 15-item geriatric depression scale (GDS) for depression, the apathy scale (AS) for apathy, and Abe's BPSD score (ABS) ${ }^{11}$. In addition, the activities of daily living (ADL) were assessed using the Alzheimer's Disease Cooperative Study-Activities of Daily Living (ADCS-ADL). These scores were assessed at the baseline and at 3, 6, 
and 12 months (M) after initiation (12 $\mathrm{M}$ as a whole).

Ethical permission for this study was provided by the Ethics Committee on

Epidemiological Studies of the Okayama University Graduate School of Medicine, Dentistry and Pharmaceutical Sciences (approval \#775), and written informed consent was obtained from all participants prior to enrollment.

\section{Statistical analysis}

Demographic and clinical data are presented as mean \pm S.D. in the text and tables.

Statistical analyses were performed using statistical software (SPSS 22.0.0.0; IBM, Armonk, New

York, USA). Differences in the distribution of demographic factors among PVH grades (0 - III) were

analyzed using the $\chi^{2}$ test and Jonckheere-Terpstra's test. After having checked for normality, baseline age, $\mathrm{Z}$ scores of VSRAD and six clinical scores (i.e., MMSE, HDS-R, GDS, AS, ABS, and ADCS-ADL) were compared using the Kraskal-Wallis test among PVH grades (0 - III) or anti-dementia drugs. Similarly, changes from the baseline at each time point were compared using the Kraskal-Wallis test among anti-dementia drugs. P-values $<0.05$ were considered to be significantly different. 


\section{Results}

\section{Participants' characteristics}

The baseline demographic and clinical features of all AD patients $(n=551)$ and each group according to the grade of PVH (0-III) are shown in Table 1. The ratio of females among the $551 \mathrm{AD}$ patients was $63.5 \%$ and the average age of all $\mathrm{AD}$ patients was $78.4 \pm 7.3$ years old (yo, mean $\pm \mathrm{SD}$ ). The number of patients in each PVH groups was as follows: PVH 0; $n=41$, PVH I; $n=212$, PVH II; $\mathrm{n}=179$, PVH III; $\mathrm{n}=119$. Although there were no differences in the gender ratio, $\mathrm{Z}$ score of VSRAD, emotional scores (GDS, AS, ABS) or ADL in the four PVH subgroups, the baseline age showed a WM-severity-dependent increase: $72.2 \pm 8.1$ yo for PVH $0,77.5 \pm 6.8$ yo for PVH I, $79.6 \pm 6.7$ yo for PVH II, and $80.5 \pm 7.4$ yo for PVH III $(* * \mathrm{p}<0.01$ vs PVH 0 , \#\# $\mathrm{p}<0.01$ vs PVH I, and $\mathrm{p}$-value for increasing trend $<0.001)$. In contrast, cognitive scores showed a WM-severity-dependent decreases in MMSE $(21.1 \pm 4.2$ for PVH $0,21.0 \pm 4.6$ for PVH I, $19.4 \pm 5.3$ for PVH II, and 19.2 \pm 4.6 for PVH III), and HDS-R (19.2 \pm 5.6 for PVH 0, $19.0 \pm 5.7$ for PVH I, $16.7 \pm 6.2$ for PVH II, and $16.7 \pm 5.3$ for PVH III) (\# p < 0.05 vs PVH I, \#\# p < 0.01 vs PVH I, and $\mathrm{p}$-value for increasing trend $<0.001$ ).

Table 2 shows the change of each drug dosage (mg/day) from 3 to $12 \mathrm{M}$, and baseline PVH-severity-dependent features of AD patients in the four drug subgroups. The average drug dosage 
showed a progressive increase from 3 to $12 \mathrm{M}$ in all four drugs (Table 2, top). Finally, 249 patients dropped out or were lost to follow up, and 302 patients remained. The number of patients in each drug subgroup was showed in Table 2 (the digit in parentheses shows the number of patients in each term). In the PVH 0 subgroup $(n=41)$, the rivastigmine group showed a higher baseline ABS $(12.3 \pm 10.0)$ than the donepezil group $(2.1 \pm 3.1, * \mathrm{p}<0.05)$. In the PVH I subgroup $(\mathrm{n}=212)$, the memantine group showed a higher baseline ABS $(9.8 \pm 6.9)$ than the donepezil group $(2.8 \pm 3.9, * \mathrm{p}<0.05)$ and the galantamine group $(3.2 \pm 4.4, \# \mathrm{p}<0.05)$. In the PVH II subgroup $(\mathrm{n}=179)$, the memantine group $(12.0 \pm 9.3)$ again showed a higher baseline ABS $(12.0 \pm 9.3)$ than the donepezil group $(3.1 \pm 5.4, * p$ $<0.05)$. There were no significant differences in the baseline age and other baseline scores.

\section{Changes from the baseline after the therapy}

Fig. 1 - 4 summarize the mean changes of each drug from the baseline (0 M) to 3, 6, 9, and

$12 \mathrm{M}$ in PVH 0-III. The data of memantine was omitted from Fig. 1 because there were only two cases

(Table 2). As shown in Fig. 1, the PVH 0 subgroup preserved cognitive MMSE and HDS-R, affective GDS, AS, ABS, and ADL until $12 \mathrm{M}$ of each drug treatment. An inter-drug comparison showed that MMSE was better preserved in the galantamine group $\left(1.1 \pm 4.0,{ }^{*} \mathrm{p}<0.05\right.$ vs donepezil) than in the donepezil group $(-1.2 \pm 1.9)$ at $9 \mathrm{M}$, while GDS was better preserved in the galantamine group $(-0.8 \pm$ 
3.9) than in the rivastigmine group $(1.2 \pm 1.6, \# \mathrm{p}<0.05$ vs galantamine $)$ at $9 \mathrm{M}$.

Fig. 2 also shows the preservation of cognitive MMSE and HDS-R, and affective GDS and

ABS until $12 \mathrm{M}$ in the PVH I subgroup. AS showed an improvement from the baseline in the

memantine group at $3 \mathrm{M}(-9.0 \pm 10.8, \uparrow \mathrm{p}<0.05)$ and $9 \mathrm{M}(-4.2 \pm 5.9,9 \mathrm{p}<0.05)$ and in the

galantamine group at $9 \mathrm{M}(-2.5 \pm 6.7, \S \S \mathrm{p}<0.01)$. The donepezil group worsened in AS at $9 \mathrm{M}$ from the baseline $(4.3 \pm 10.0, \uparrow \mathrm{p}<0.05)$. ADL worsened in the galantamine group $(-0.9 \pm 4.0, \S \mathrm{p}<0.05)$

at $12 \mathrm{M}$. The inter-drug comparison showed an improvement of ABS in the memantine group $(-5.4 \pm$ $4.2,{ }^{*} \mathrm{p}<0.05$ vs donepezil) than in the donepezil group $(0.4 \pm 2.6)$ at $9 \mathrm{M}$.

Fig. 3 shows well preserved affective GDS, AS, and ABS until $12 \mathrm{M}$ in the PVH II subgroup. MMSE showed a significant improvement from the baseline in the galantamine group (1.5 $\pm 2.2, \S \mathrm{p}<0.05)$ at $9 \mathrm{M}$ and HDS-R $(0.4 \pm 3.7, \S \mathrm{p}<0.05)$ at $3 \mathrm{M}$. However, the galantamine group showed a significant decline of ADL $(-1.8 \pm 2.6, \S \mathrm{p}<0.05)$ at $12 \mathrm{M}$. The inter-drug comparison showed better preservation of MMSE in the galantamine group $(1.5 \pm 2.2, * * p<0.01$ vs donepezil $)$ than in the donepezil group $(-2.8 \pm 2.4)$ at $9 \mathrm{M}$.

As shown in Fig. 4, the PVH III subgroup showed well preserved MMSE, HDS-R, GDS, $\mathrm{AS}$, and ABS until $12 \mathrm{M}$. ADL deteriorated in the riverstigmine group at $6 \mathrm{M}(-3.3 \pm 2.5, \pm \mathrm{p}<0.05)$ and $12 \mathrm{M}(-3.3 \pm 2.7, \pm \mathrm{p}<0.05)$. The inter-drug comparison showed better preservation of HDS-R in 
the rivastigmine group $(0.5 \pm 4.5, * * \mathrm{p}<0.01$ vs donepezil $)$ and the galantamine group $(-0.5 \pm 5.4, * \mathrm{p}$

$<0.05$ vs donepezil) than in the donepezil group $(-4.1 \pm 4.6)$.

\section{Discussion}

In the present study, we evaluated the clinical features of $\mathrm{AD}$ patients who were divided into four subgroups according to the PVH grade (0-III). The baseline age became higher with PVH grades (Table 1), and cognitive scores (MMSE and HDS-R) showed a WM-severity-dependent decrease (Table 1). The ABS baseline of the rivastigmine group (PVH 0) and the memantine group (PVH I-II) was significantly higher than that of the donepezil group, while that of the memantine group was significantly higher than that of the galantamine group (Table 2). However, there were no significant differences in the baseline age and other baseline scores among the four drug groups (Table 2). The PVH 0 subgroup showed preserved cognitive, affective, and ADL functions until $12 \mathrm{M}$ in all four drug groups. After treatment with memantine and galantamine, AS improved significantly from the baseline in the PVH I subgroup while galantamine showed a worse ADL from the baseline (Fig. 2).

In the PVH II subgroup, galantamine showed a significant improvement of cognitive scores (MMSE and HDS-R) from the baseline but a worse ADL from the baseline (Fig. 3). In the PVH III subgroup, 
MMSE, HDS-R, GDS, AS, and ABS were well preserved in all four drug groups until $12 \mathrm{M}$, but ADL deteriorated in the riverstigmine group at 6 and $12 \mathrm{M}$ (Fig. 4).

Previous studies indicated that WMLs such as PVH and DWMH reflect demyelination, loss of ependymal cells, arteriosclerosis, and microinfarcts. PVH was associated with a loss of ventricular lining, and DWMH with deep WM demyelination ${ }^{12-14}$. The volume of PVH was also associated with neurofibrillary tangles, indicating that the mechanism of AD may contribute to the vascular or white matter pathology ${ }^{4}$. Our previous studies demonstrated that the severity of WMLs was closely related to a deterioration of the cognitive and affective functions in $\mathrm{AD}$ patients ${ }^{5,6}$. Moreover, $\mathrm{PVH}$ was related to a decline in cognitive function ${ }^{15}$ and mental processing speed ${ }^{16}$. In the present study, cognitive scores (MMSE and HDS-R) showed a WM-severity-dependent decrease with aging (Table 1), suggesting that a worse PVH may reflect progression of AD pathology.

The present study first described the clinical effect of four anti-dementia drugs on AD patients who were divided into four PVH-severity-dependent subgroups. The positive effect of galantamine in the PVH I-II subgroups may be consistent with a previous report, where galantamine was effective on cognitive, affective and ADL functions of AD accompanied by cerebrovascular disease ${ }^{17}$ with increasing vascular reactivity ${ }^{18}$ and regional cerebral blood flow ${ }^{19}$. We have also previously reported that galantamine improved frontal lobe cognitive function in elderly AD patients 
${ }^{20}$, and maintained or improved cognitive, affective and ADL functions in AD with asymptomatic lacunar infarction ${ }^{21}$.

There are several reports that showed that donepezil was effective for both positive and negative affective symptoms ${ }^{22-24}$. However, our present results showed that AS deteriorated at $9 \mathrm{M}$ in the PVH I subgroup (Fig. 2). Similarly, rivastigmine was reported to be effective for cognitive and ADL functions in severe AD patients ${ }^{25}$. However, our present results showed a deterioration of ADL at 6 and $12 \mathrm{M}$ in the PVH III subgroup (Fig. 4). These discrepancies may be due to different severities of AD patients between previous and the present study and our PVH-severity-dependent analysis.

Some reports showed significant effects of memantine in cognitive severe impairment battery (SIB) and ADCS-ADL for moderate to severe AD patients ${ }^{15,26}$. In agreement with our previous results ${ }^{27,28}$, the present study showed a significant improvement from the baseline in AS of the PVH I subgroup at 3 and $9 \mathrm{M}$ (Fig. 2).

There are limitations to our retrospective study, considering nature of $\mathrm{AD}$ treatment, outcome of measurements would be evaluated in the longest observation time. In other words, different transient effect in one time point seems to be difficult to evaluate.

In conclusion, the present study demonstrated that cognitive scores (MMSE and HDS-R) showed a WM-severity-dependent decreases with increasing age (Table 1). Moreover, the four drugs 
showed WM-severity-dependent sensitivity when assessing the clinical effect of cognitive, affective and ADL functions.

\section{Acknowledgments}

This work was partly supported by Grants-in-Aid for Scientific Research (B, 25293202), (C, 15K09316), Challenging Research (15K15527) and Young Research (15K21181), and by

Grants-in-Aid from the Research Committees (Mizusawa H, Nakashima K, Nishizawa M, Sasaki H, and Aoki M) from the Ministry of Health, Labour and Welfare of Japan.

\section{Conflict of interest}

Dr. K. Abe received an honorarium from Takeda Pharmaceutical Company Limited, but other authors declare no financial or other conflicts of interest.

\section{References}

[1] Lee S, Viqar F, Zimmerman ME, et al. White matter hyperintensities are 
a core feature of Alzheimer's disease: Evidence from the dominantly inherited Alzheimer network. Annals of neurology 2016; 79: 929-39.

[2] Murray AD, Staff RT, Shenkin SD, Deary IJ, Starr JM, Whalley LJ. Brain white matter hyperintensities: relative importance of vascular risk factors in nondemented elderly people. Radiology 2005; 237 : 251-7.

[3] Brickman AM. Contemplating Alzheimer's disease and the contribution of white matter hyperintensities. Current neurology and neuroscience reports $2013 ; 13: 415$.

[4] Shim YS, Yang DW, Roe CM, et al. Pathological correlates of white matter hyperintensities on magnetic resonance imaging. Dementia and geriatric cognitive disorders 2015; 39: 92-104.

[5] Hishikawa N, Fukui Y, Sato K, et al. Cognitive and affective functions in Alzheimer's disease patients with metabolic syndrome. European journal of neurology 2016; 23: 339-45.

[6] Tokuchi R, Hishikawa N, Sato K, et al. Age-dependent cognitive and affective differences in Alzheimer's and Parkinson's diseases in relation to MRI findings. Journal of the neurological sciences 2016; 365:3-8.

[7] Burns JM, Church JA, Johnson DK, et al. White matter lesions are prevalent but differentially related with cognition in aging and early Alzheimer disease. Archives of neurology 2005; 62: 1870-6.

[8] Fazekas F, Chawluk JB, Alavi A, Hurtig HI, Zimmerman RA. MR signal abnormalities at $1.5 \mathrm{~T}$ in Alzheimer's dementia and normal aging. AJR American journal of roentgenology 1987; 149: 351-6.

[9] Hirata Y, Matsuda H, Nemoto K, et al. Voxel-based morphometry to discriminate early Alzheimer's disease from controls. Neuroscience letters 2005; 382: 269-74.

[10] Li X, Shimizu S, Jibiki I, Watanabe K, Kubota T. Correlations between Z-scores of VSRAD and regional cerebral blood flow of SPECT in patients with Alzheimer's disease and mild cognitive impairment. Psychiatry and clinical neurosciences 2010; 64: 284-92.

[11] Abe K, Yamashita T, Hishikawa N, et al. A new simple score (ABS) for assessing behavioral and psychological symptoms of dementia. Journal of the neurological sciences 2015; 350: 14-7. 
[12] Sze G, De Armond SJ, Brant-Zawadzki M, Davis RL, Norman D, Newton TH. Foci of MRI signal (pseudo lesions) anterior to the frontal horns: histologic correlations of a normal finding. AJR American journal of roentgenology 1986; 147: $331-7$.

[13] Revesz T, Hawkins CP, du Boulay EP, Barnard RO, McDonald WI. Pathological findings correlated with magnetic resonance imaging in subcortical arteriosclerotic encephalopathy (Binswanger's disease). Journal of neurology, neurosurgery, and psychiatry 1989; 52: 1337-44.

[14] Fazekas F, Kleinert R, Offenbacher H, et al. Pathologic correlates of incidental MRI white matter signal hyperintensities. Neurology 1993; 43: 1683-9. [15] van Dyck CH, Tariot PN, Meyers B, Malca Resnick E, Memantine MEMMDSG. A 24-week randomized, controlled trial of memantine in patients with moderate-to-severe Alzheimer disease. Alzheimer disease and associated disorders 2007; 21: 136-43.

[16] van den Heuvel DM, ten Dam VH, de Craen AJ, et al. Increase in periventricular white matter hyperintensities parallels decline in mental processing speed in a non-demented elderly population. Journal of neurology, neurosurgery, and psychiatry 2006; 77: 149-53.

[17] Erkinjuntti T, Gauthier S, Bullock R, et al. Galantamine treatment in Alzheimer's disease with cerebrovascular disease: responder analyses from a randomized, controlled trial (GAL-INT-6). Journal of psychopharmacology 2008; 22: $761-8$.

[18] Bar KJ, Boettger MK, Seidler N, Mentzel HJ, Terborg C, Sauer H. Influence of galantamine on vasomotor reactivity in Alzheimer's disease and vascular dementia due to cerebral microangiopathy. Stroke; a journal of cerebral circulation 2007; 38: 3186-92.

[19] Shimizu S, Kanetaka H, Hirose D, Sakurai H, Hanyu H. Differential effects of acetylcholinesterase inhibitors on clinical responses and cerebral blood flow changes in patients with Alzheimer's disease: a 12-month, randomized, and open-label trial. Dementia and geriatric cognitive disorders extra 2015; 5: 135-46. [20] Nakano Y, Matsuzono K, Yamashita T, et al. Long-Term Efficacy of Galantamine in Alzheimer's Disease: The Okayama Galantamine Study (OGS). Journal of Alzheimer's disease : JAD 2015; 47: 609-17. 
[21] Hishikawa N, Fukui Y, Sato K, Ohta Y, Yamashita T, Abe K.

Comprehensive effects of galantamine and cilostazol combination therapy on patients with Alzheimer's disease with asymptomatic lacunar infarction.

Geriatrics \& gerontology international 2016; In press.

[22] Gauthier S, Feldman H, Hecker J, et al. Efficacy of donepezil on behavioral symptoms in patients with moderate to severe Alzheimer's disease. International psychogeriatrics / IPA 2002; 14: 389-404.

[23] Holmes C, Wilkinson D, Dean C, et al. The efficacy of donepezil in the treatment of neuropsychiatric symptoms in Alzheimer disease. Neurology 2004; 63: $214-9$.

[24] Kavirajan H, Schneider LS. Efficacy and adverse effects of cholinesterase inhibitors and memantine in vascular dementia: a meta-analysis of randomised controlled trials. The Lancet Neurology 2007; 6: 782-92.

[25] Farlow MR, Grossberg G, Gauthier S, Meng X, Olin JT. The ACTION study: methodology of a trial to evaluate safety and efficacy of a higher dose rivastigmine transdermal patch in severe Alzheimer's disease. Current medical research and opinion 2010; 26: 2441-7.

[26] Reisberg B, Doody R, Stoffler A, et al. Memantine in moderate-to-severe Alzheimer's disease. The New England journal of medicine 2003; 348: 1333-41. [27] Matsuzono K, Hishikawa N, Ohta Y, et al. Combination Therapy of Cholinesterase Inhibitor (Donepezil or Galantamine) plus Memantine in the Okayama Memantine Study. Journal of Alzheimer's disease : JAD 2015; 45: 771-80.

[28] Matsuzono K, Yamashita T, Ohta Y, et al. Clinical Benefits of Memantine Treatment for Alzheimer's Disease in the Okayama Memantine Study II (OMS II). Journal of Alzheimer's disease : JAD 2015; 47: 487-93.

\section{Figure legends}


Fig. 1) The mean changes of clinical scores in each drug (donepezil, galantamine, and rivastigmine) from the baseline (0 M) to 3, 6, 9, and $12 \mathrm{M}$ in the PVH 0 subgroup. Cognitive MMSE and HDS-R, affective GDS, AS, ABS, and ADL were well preserved until $12 \mathrm{M}$ of each drug treatment.

Fig. 2) The mean changes of clinical scores in each drug (donepezil, galantamine, rivastigmine, and memantine) from the baseline (0 M) to 3, 6, 9, and $12 \mathrm{M}$ in the PVH I subgroup. AS showed improvements from the baseline in response to memantine at 3 and $9 \mathrm{M}(\boldsymbol{q} \mathrm{p}<0.05)$ and galantamine at $9 \mathrm{M}(\S \S \mathrm{p}<0.01)$.

Fig. 3) The mean changes of clinical scores in each drug (donepezil, galantamine, rivastigmine, and memantine) from the baseline (0 M) to 3, 6, 9, and $12 \mathrm{M}$ in the PVH II subgroup. MMSE showed a significant improvement from the baseline in response to galantamine $(\S p<0.05)$ at $9 \mathrm{M}$ and HDS-R $(\S \mathrm{p}<0.05)$ at $3 \mathrm{M}$.

Fig. 4) The mean changes of clinical scores in each drug (donepezil, galantamine, rivastigmine, and memantine) from the baseline ( $0 \mathrm{M})$ to 3, 6, 9, and $12 \mathrm{M}$ in the PVH III subgroup. ADL deteriorated in the riverstigmine group at 6 and $12 \mathrm{M}(\$ \mathrm{p}<0.05)$. 
Fig. 1

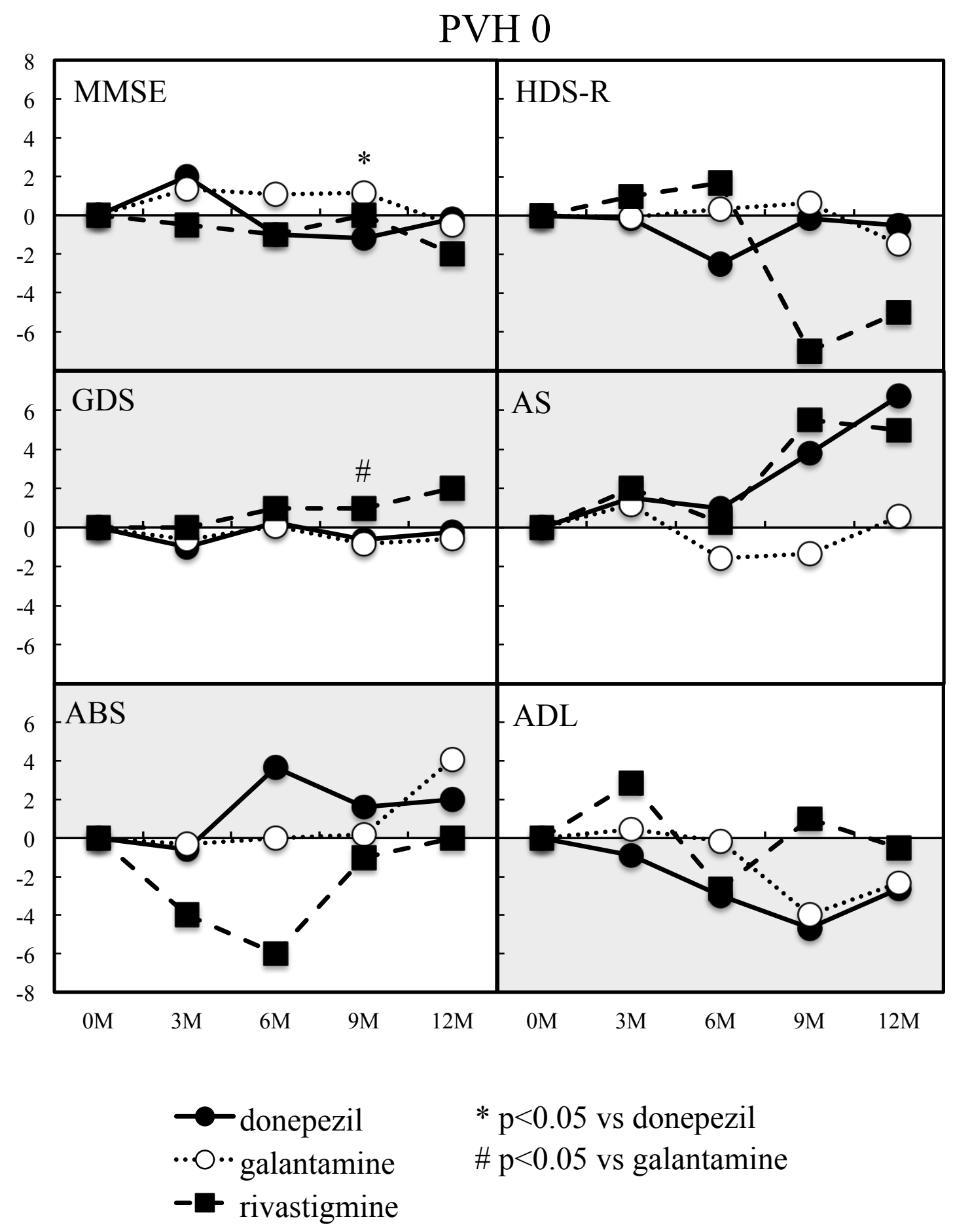


Fig. 2

\section{PVH I}

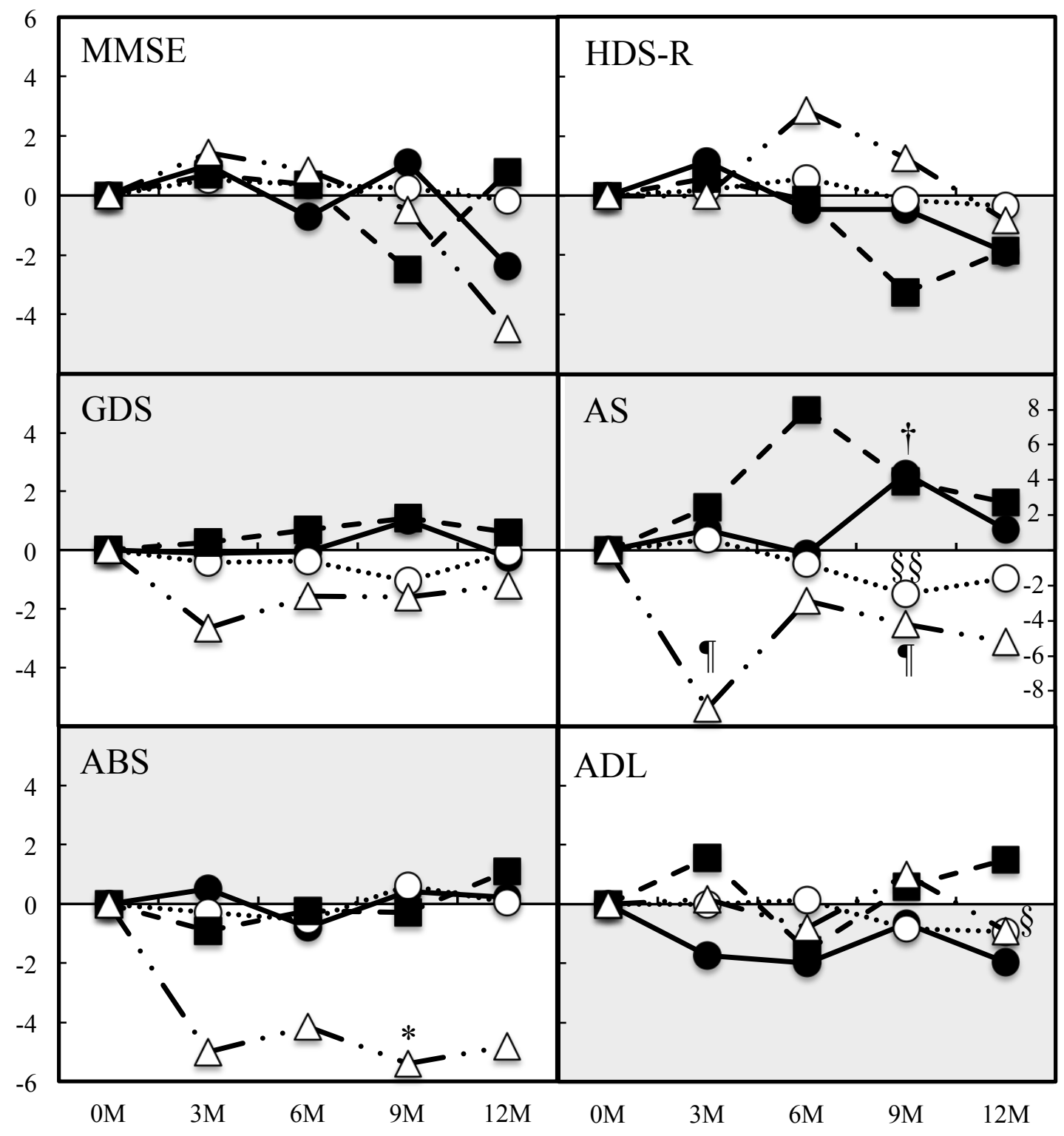

$\rightarrow$ donepezil

.. -. galantamine

- r rivastigmine

$\rightarrow \cdot$ memantine $\dagger \mathrm{p}<0.05$ vs baseline donepezil

$\S \mathrm{p}<0.05$ vs baseline galantamine $\S \S p<0.01$ vs baseline galantamine If $\mathrm{p}<0.05$ vs baseline memantine

* $\mathrm{p}<0.05$ vs donepezil 
Fig. 3

PVH II

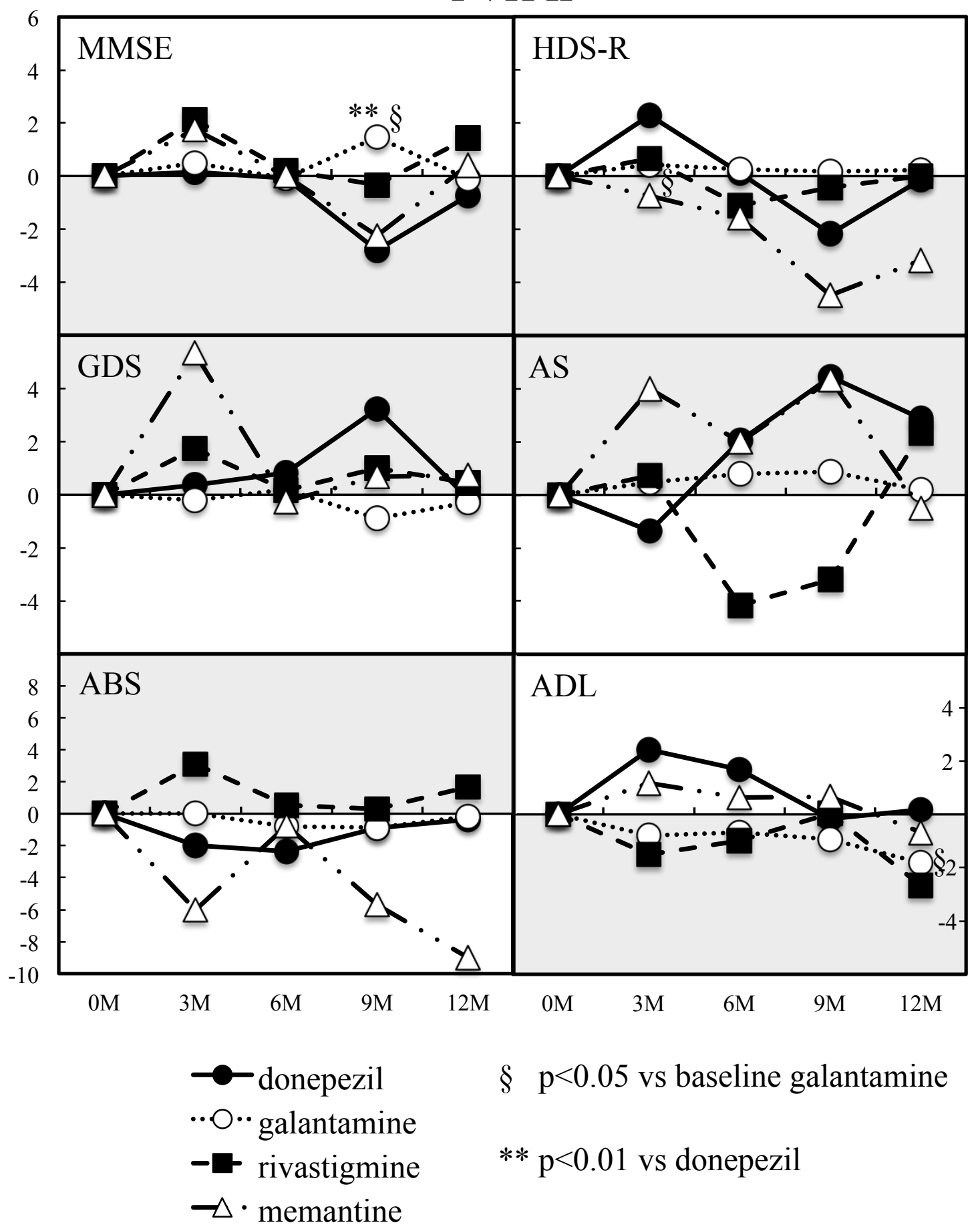


Fig. 4

\section{PVH III}

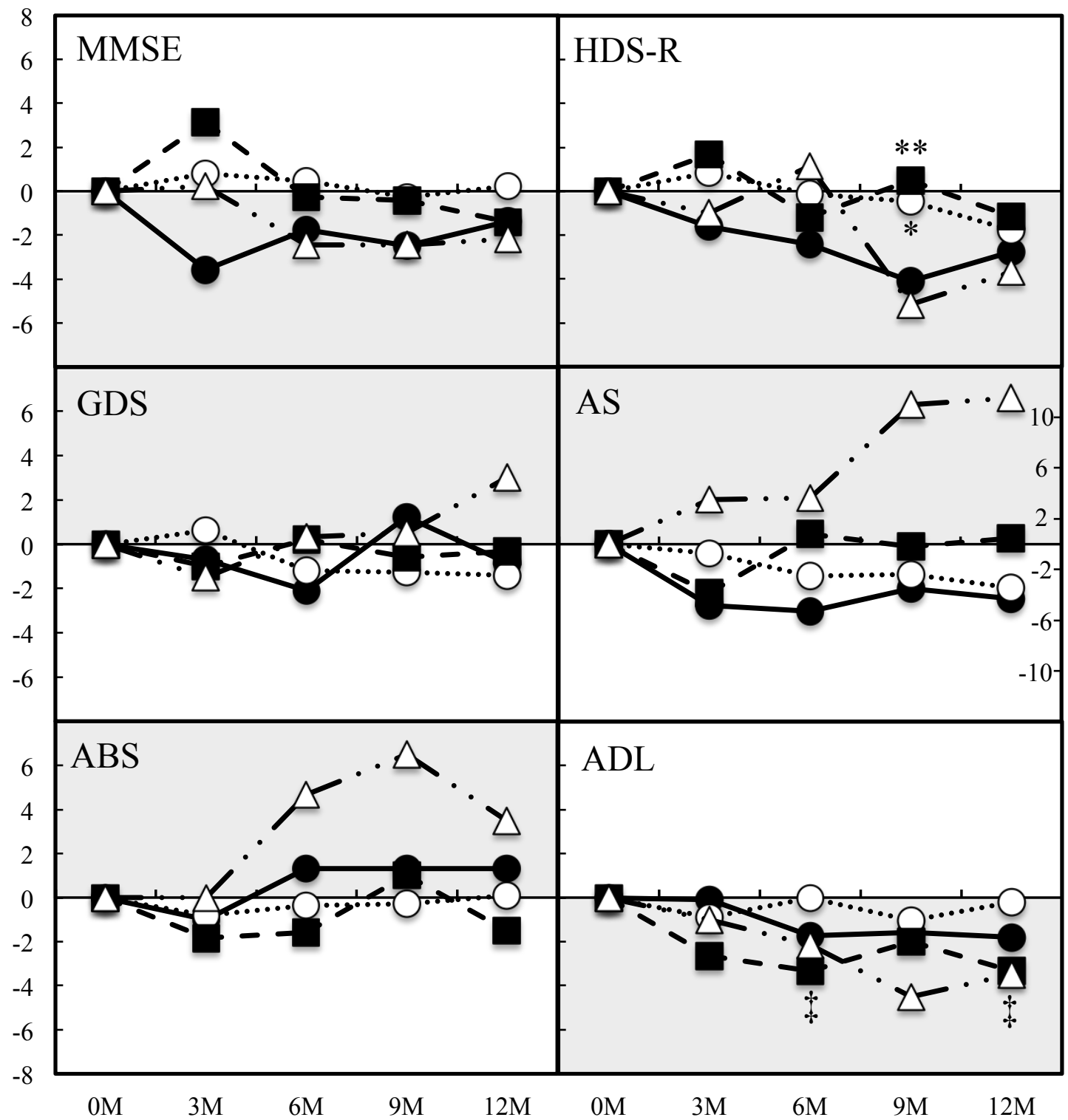
$\longrightarrow$ donepezil
$\ddagger \mathrm{p}<0.05$ vs baseline rivastigmine
*.* galantamine
- - r rivastigmine
* $\mathrm{p}<0.05$ vs donepezil
$\rightarrow \cdot$ memantine
$* * p<0.01$ vs donepezil 
Table 1

Baseline demographic and clinical characteristics of all AD patients and each subgroup according to Fazekas scale

\begin{tabular}{|c|c|c|c|c|c|c|c|c|c|c|}
\hline & $\begin{array}{l}\text { all AD } \\
\text { patients }\end{array}$ & PVH 0 & PVH I & & PVH II & & PVH III & & p-value & $\begin{array}{l}\mathrm{p} \text {-value } \\
\text { for trend }\end{array}$ \\
\hline $\mathrm{n}$ & 551 & 41 & 212 & & 179 & & 119 & & & \\
\hline Gender (male/female) & $201 / 350$ & $18 / 23$ & $83 / 129$ & & $52 / 127$ & & $48 / 71$ & & & \\
\hline Female $(\%)$ & 63.5 & 56.1 & 60.8 & & 70.9 & & 59.7 & & $0.084^{\mathrm{a}}$ & \\
\hline Age (years) & $78.4 \pm 7.3$ & $72.2 \pm 8.1$ & $77.5 \pm 6.8$ & $* *$ & $79.6 \pm 6.7$ & \#\# & $80.5 \pm 7.4$ & \#\# & $<0.001^{\mathrm{b}}$ & $0.001^{\mathrm{c}}$ \\
\hline VSRAD & $2.7 \pm 1.3$ & $2.6 \pm 1.3$ & $2.5 \pm 1.1$ & & $2.7 \pm 1.1$ & & $2.9 \pm 1.6$ & & & \\
\hline \multicolumn{11}{|l|}{ Baseline score } \\
\hline MMSE & $20.1 \pm 4.9$ & $21.1 \pm 4.2$ & $21.0 \pm 4.6$ & & $19.4 \pm 5.3$ & $\#$ & $19.2 \pm 4.6$ & \# & $0.002^{\mathrm{b}}$ & $0.001^{\mathrm{c}}$ \\
\hline HDS-R & $17.8 \pm 5.9$ & $19.2 \pm 5.6$ & $19.0 \pm 5.7$ & & $16.7 \pm 6.2$ & \#\# & $16.7 \pm 5.3$ & \#\# & $<0.001^{\mathrm{b}}$ & $0.001^{\mathrm{c}}$ \\
\hline GDS & $5.3 \pm 3.8$ & $4.6 \pm 3.7$ & $5.1 \pm 3.7$ & & $5.4 \pm 3.8$ & & $6.0 \pm 4.1$ & & $0.273^{\mathrm{b}}$ & \\
\hline AS & $16.8 \pm 9.4$ & $15.1 \pm 8.4$ & $16.4 \pm 9.7$ & & $16.9 \pm 9.0$ & & $18.5 \pm 9.5$ & & $0.226^{\mathrm{b}}$ & \\
\hline ABS & $4.0 \pm 5.5$ & $4.6 \pm 6.3$ & $3.8 \pm 5.2$ & & $3.9 \pm 5.7$ & & $4.4 \pm 5.6$ & & $0.706^{\mathrm{b}}$ & \\
\hline ADL & $20.9 \pm 5.8$ & $21.6 \pm 6.3$ & $21.3 \pm 5.7$ & & $20.4 \pm 5.8$ & & $20.5 \pm 5.9$ & & $0.442^{\mathrm{b}}$ & \\
\hline
\end{tabular}
a: $\chi^{2}$ test
b: Kruskal-Wallis test
c: Jonkheere-Terpstra test

** $\mathrm{p}<0.01$ vs PVH 0 group

$\# \mathrm{p}<0.05$ vs PVH I group

$\# \#$ p $<0.01$ vs PVH I group 
Table 2

Change of drug dosage and baseline demographic and clinical characteristics of AD patients

\begin{tabular}{|c|c|c|c|c|c|c|}
\hline \multirow[b]{2}{*}{ Dosage (mg/day) } & donepezil & galantamine & rivastigmine & memantine & & \multirow[t]{2}{*}{ p-value } \\
\hline & & & & & & \\
\hline 3 months & 5.5 & 13.7 & $10.9 \pm$ & 13.8 & & \\
\hline 6 months & 6.2 & $15.6 \pm$ & $12.3 \pm 5.6$ & 16.0 & & \\
\hline 12 months & $6.7 \pm 2.4$ & $17.2 \pm 5.4$ & $13.9 \pm 5.3$ & $17.3 \pm 4.4$ & & \\
\hline \multicolumn{7}{|l|}{ PVH 0} \\
\hline Female $(\%)$ & 81.8 & 58.3 & 25.0 & 0 & & \\
\hline Age (years) & $69.9 \pm 7.7$ & $73.0 \pm 8.8$ & $71.3 \pm 2.6$ & 78.0 & & 0.618 \\
\hline \multicolumn{7}{|l|}{ Baseline score } \\
\hline MMSE & $21.4 \pm 4.3$ & $20.4 \pm 4.1$ & $21.8 \pm 1.3$ & 27.0 & & 0.704 \\
\hline HDS-R & $20.3 \pm 5.4$ & $18.1 \pm 5.6$ & $20.0 \pm 4.8$ & 24.0 & & 0.569 \\
\hline \multicolumn{7}{|l|}{ PVH I } \\
\hline $\mathrm{n}(3 \mathrm{M}, 6 \mathrm{M}, 9 \mathrm{M}, 12 \mathrm{M})$ & $47(47,36,32,29)$ & $133(133,124,106,84)$ & $19(19,1714,12)$ & $13(13,9,7,6)$ & & \\
\hline Gender (male/female) & $16 / 31$ & $52 / 81$ & $10 / 9$ & $5 / 8$ & & \\
\hline Female $(\%)$ & 66.0 & 60.9 & 47.4 & 61.5 & & \\
\hline Age (years) & $77.8 \pm 6.5$ & $77.3 \pm 7.0$ & $76.8 \pm 4.7$ & $78.5 \pm 7.7$ & & 0.664 \\
\hline \multicolumn{7}{|l|}{ Baseline score } \\
\hline MMSE & $21.9 \pm 4.3$ & $21.0 \pm 4.4$ & $20.3 \pm 5.8$ & $18.5 \pm 4.8$ & & 0.207 \\
\hline HDS-R & $19.5 \pm 6.4$ & $19.2 \pm 5.1$ & $19.3 \pm 6.9$ & $16.2 \pm 5.9$ & & 0.268 \\
\hline GDS & $5.0 \pm 3.4$ & $5.2 \pm 3.9$ & $3.9 \pm 2.9$ & $6.1 \pm 3.4$ & & 0.488 \\
\hline AS & $15.2 \pm 7.3$ & $16.8 \pm 10.1$ & $14.4 \pm 7.2$ & $19.0 \pm 13.3$ & & 0.845 \\
\hline \multicolumn{7}{|l|}{ Baseline score } \\
\hline MMSE & $19.7 \pm 6.0$ & $19.7 \pm 4.9$ & $17.3 \pm 5.1$ & $21.3 \pm 4.0$ & & 0.051 \\
\hline HDS-R & $16.1 \pm 7.2$ & $17.4 \pm 5.8$ & $15.0 \pm 6.2$ & $17.8 \pm 4.6$ & & 0.263 \\
\hline GDS & $5.4 \pm 3.7$ & $5.1 \pm 4.1$ & $6.4 \pm 3.2$ & $6.5 \pm 2.8$ & & 0.414 \\
\hline AS & $14.5 \pm 7.9$ & $16.6 \pm 8.9$ & $20.1 \pm 8.7$ & $21.5 \pm 9.7$ & & 0.104 \\
\hline ABS & $3.1 \pm 5.4$ & $3.5 \pm 5.3$ & $4.3 \pm 4.2$ & $12.0 \pm 9.3$ & $*$ & $<0.05$ \\
\hline $\mathrm{ADL}$ & $19.9 \pm 5.3$ & $20.9 \pm 6.3$ & $19.4 \pm 3.8$ & $19.3 \pm 7.0$ & & 0.362 \\
\hline \multicolumn{7}{|l|}{ PVH III } \\
\hline $\mathrm{n}(3 \mathrm{M}, 6 \mathrm{M}, 9 \mathrm{M}, 12 \mathrm{M})$ & $22(22,18,15,8)$ & $69(69,58,51,30)$ & $19(19,15,12,6)$ & $9(9,9,7,6)$ & & \\
\hline Gender (male/female) & $7 / 15$ & $30 / 39$ & $8 / 11$ & $3 / 6$ & & \\
\hline Female $(\%)$ & 68.2 & 56.5 & 57.9 & 66.7 & & \\
\hline Age (years) & $80.3 \pm 5.4$ & $79.7 \pm 8.9$ & $82.8 \pm 3.4$ & $81.6 \pm 4.5$ & & 0.283 \\
\hline \multicolumn{7}{|l|}{ Baseline score } \\
\hline MMSE & $17.4 \pm 5.5$ & $20.0 \pm 4.2$ & $18.3 \pm 4.7$ & $20.1 \pm 3.3$ & & 0.235 \\
\hline HDS-R & $14.5 \pm 5.7$ & $17.7 \pm 4.9$ & $15.6 \pm 6.0$ & $16.2 \pm 3.8$ & & 0.196 \\
\hline GDS & $6.0 \pm 4.4$ & $5.7 \pm 3.9$ & $8.9 \pm 3.6$ & $5.7 \pm 3.7$ & & 0.316 \\
\hline AS & $18.6 \pm 10.2$ & $17.8 \pm 9.2$ & $25.9 \pm 8.5$ & $13.0 \pm 4.3$ & & 0.135 \\
\hline ABS & $4.8 \pm 6.5$ & $3.9 \pm 5.2$ & $7.6 \pm 6.4$ & $3.3 \pm 3.4$ & & 0.155 \\
\hline $\mathrm{ADL}$ & $20.5 \pm 5.8$ & $21.1 \pm 5.1$ & $18.9 \pm 8.1$ & $16.8 \pm 7.2$ & & 0.770 \\
\hline
\end{tabular}

